\title{
Cooling and trapping of ultracold strontium isotopic mixtures
}

\author{
N. Poli, R. E. Drullinger, G. Ferrari, J. Léonard, * F. Sorrentino, and G. M. Tino ${ }^{\dagger}$ \\ Dipartimento di Fisica—LENS, Università di Firenze, Istituto Nazionale di Fisica Nucleare-Sezione di Firenze, \\ Istituto Nazionale di Fisica della Materia-Unità di Firenze, \\ Polo Scientifico, 50019 Sesto Fiorentino, Italy
}

(Received 8 March 2005; published 13 June 2005)

\begin{abstract}
We present the simultaneous cooling and trapping of an isotopic mixture in a magneto-optical trap and describe the transfer of the mixture into a conservative, far-off-resonant dipole trap. The mixture is prepared with a technique that applies to intermediate and heavy alkaline-earth-metal-like atoms. In this work, ${ }^{88} \mathrm{Sr}$ and ${ }^{86} \mathrm{Sr}$ are simultaneously loaded first into the magneto-optical trap operated on the ${ }^{1} S_{0^{-}}{ }^{3} P_{1}$ spin-forbidden line at $689 \mathrm{~nm}$, and then transferred into the dipole trap. We observe fast interspecies thermalization in the dipole trap which allows one to set a lower bound on the ${ }^{88} \mathrm{Sr}-{ }^{86} \mathrm{Sr}$ elastic cross section.
\end{abstract}

DOI: 10.1103/PhysRevA.71.061403

PACS number(s): 32.80.Pj, 42.62.Fi, 34.50.-s, 06.30.Ft

Recently, laser-cooled atomic strontium has been the subject of active research in various fields spanning from alloptical cooling towards quantum degeneracy for bosonic and fermionic isotopes $[1,2]$, cooling physics $[3,4]$, continuous atom laser [5], detection of ultranarrow transitions [6-9], multiple scattering [10], and collisional theory [11].

As a result of the efficient double-stage Doppler cooling, strontium is a promising candidate to reach quantum degeneracy. Second-stage Doppler cooling [1] working on the ${ }^{1} S_{0^{-}}{ }^{3} P_{1}$ intercombination transition (see Fig. 1) combined with optical dipole trapping was proven effective to directly reach phase-space densities of $\rho=0.1$ [12]. Further increase of $\rho$ seems to be limited by light-assisted collisions and direct evaporative cooling was not reported so far. Alternative cooling techniques are offered by mixtures of different atomic species [13] or different isotopes [14,15]. Mixtures offer a way to exploit collisional physics not applicable in single species samples. They also offer additional degrees of freedom, such as sympathetic cooling, in order to achieve the degenerate quantum regime with atoms for which evaporative cooling is not efficient [16].

In this article we present the simultaneous cooling and trapping of isotopes of atomic strontium in a magneto-optical trap (MOT) [17] and describe the transfer of the mixture into a conservative, far-off-resonant dipole trap (FORT). The isotopic mixture is prepared with a technique based on sequentially loading different isotopes into a magnetic trapped metastable state through the leakage of a MOT operating on a single isotope. Afterwards the magnetically trapped mixture is optically pumped down to the ground state for further cooling close to the recoil limit on the intercombination ${ }^{1} S_{0^{-}}{ }^{3} P_{1}$ transition. Then the mixture is loaded into a FORT. The advantage of this method, comparing with previous works based on simultaneous optical cooling and trapping of two species $[14,15]$, is the drastic simplification of the laser source and the elimination of possible interferences between

\footnotetext{
*Present address: Van der Waals-Zeeman Institute, Amsterdam, The Netherlands. Permanent address: IPCMS, Strasbourg, France.

†Electronic address: guglielmo.tino@fi.infn.it
}

the simultaneous loading into the MOTs. For the sake of clarity we start by describing the production of a singleisotope sample, then we explain how we extend the procedure to the production of isotopic mixtures.

In the experimental setup we use a thermal source of strontium atoms as described in [8]. Strontium is evaporated in an oven heated to $500{ }^{\circ} \mathrm{C}$. The atomic beam is collimated through a bundle of capillaries which limits the geometric divergence to $50 \mathrm{mrad}$ full width. After the capillaries, the atomic beam brightness is increased by a factor of 4 with a transverse cooling stage realized with multipass beams [18] in two orthogonal planes, with a frequency offset by $20 \mathrm{MHz}$ to the red of the ${ }^{1} S_{0^{-}}{ }^{-} P_{1}$ transition $(\Gamma / 2 \pi=32 \mathrm{MHz})$. After the collimation stage, the atomic beam is slowed to a few tens of $\mathrm{m} / \mathrm{s}$ in a 30-cm-long Zeeman slower [19] based on a two-stage tapered coil with zero crossing magnetic field and a counterpropagating laser beam tuned $15 \Gamma$ to the red of the ${ }^{1} S_{0^{-}}{ }^{1} P_{1}$ transition. The light beam power is typically $18 \mathrm{~mW}$, has a $7-\mathrm{mm} e^{-2}$ radius at the MOT, and is focused on the capillaries, $85 \mathrm{~cm}$ away from the MOT. The slowed atoms are then further cooled and trapped in a MOT working on the ${ }^{1} S_{0^{-}}{ }^{1} P_{1}$ transition red detuned by $40 \mathrm{MHz}$ from the resonance. The MOT is formed by three retroreflected beams with an $e^{-2}$ radius of $5 \mathrm{~mm}$ crossing almost orthogonally,

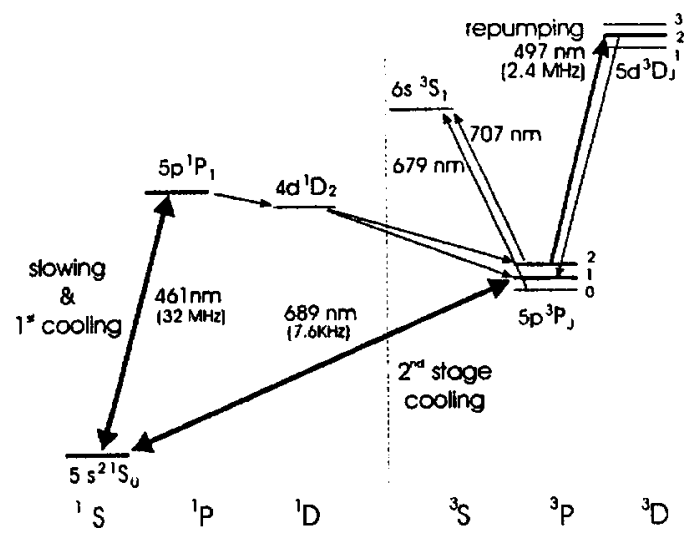

FIG. 1. Relevant energy levels and optical transitions for laser cooling and trapping of strontium. 
with the vertical beam collinear with the magnetic quadrupole axis of an anti-Helmholz pair of coils. The total $461 \mathrm{~nm}$ light intensity incident on the MOT region amounts to $46 \mathrm{~mW} / \mathrm{cm}^{2}$, and the magnetic gradient is $56 \mathrm{G} / \mathrm{cm}$. The atom number, spatial density, and temperature are derived in time of flight by imaging on a charge-coupled device (CCD) camera the atomic absorption of a $70-\mu$ s probe beam resonant on the ${ }^{1} S_{0}{ }^{-1} P_{1}$ transition. The $461-n m$ light source for the first cooling stage, as described in [20], is based on frequency doubling of a semiconductor laser. It routinely delivers $150 \mathrm{~mW}$ at $461 \mathrm{~nm}$ in a 4-MHz linewidth full width at half maximum (FWHM). The source is frequency stabilized with saturated spectroscopy on a strontium vapor cell [21].

After the initial capture in the blue MOT, and following the approach described in [1], the atoms are further cooled in a MOT operating on the ${ }^{1} S_{0}{ }^{3} P_{1}$ intercombination line at 689 $\mathrm{nm}$. The $689-\mathrm{nm}$ light is provided by a master-slave semiconductor laser system that is frequency narrowed by locking to an optical cavity which is, in turn, steered to the ${ }^{1} S_{0}{ }^{-3} P_{1}$ transition by saturation spectroscopy in a second strontium heatpipe [8]. The red beam is mode matched to the 461-nm MOT beam and is overlapped to it on a dichroic mirror. From this point the two beams share the same broadband optics up to the atoms.

As discussed in the following, the atoms leak from the blue MOT to the metastable ${ }^{3} P_{2}$ due to the $2 \times 10^{-5}$ branching ratio of the ${ }^{1} P_{1}$ excited state to the low-lying ${ }^{1} D_{2}$ and the subsequent decay to the ${ }^{3} P_{2}$ state. This loss channel is eliminated with a single optical pumping process, which involves the ${ }^{3} P_{2}{ }^{3} D_{2}$ transition at $497 \mathrm{~nm}$. For this purpose we developed a laser source based on an antireflection coated laserdiode stabilized in the Littrow extended cavity configuration, which delivers $25 \mathrm{~mW}$ at $994 \mathrm{~nm}$. This light is frequency doubled on a $17-\mathrm{mm}$-long $\mathrm{KNbO}_{3}$ crystal placed in a resonant cavity for improved conversion efficiency resulting in 4 $\mathrm{mW}$ at $497 \mathrm{~nm}$. The $\mathrm{KNbO}_{3}$ is $b$ cut for noncritical phase matching at a temperature of $54{ }^{\circ} \mathrm{C}$. The $497-\mathrm{nm}$ beam is sent to the MOT region with a $1.5-\mathrm{cm} e^{-2}$ diameter, it is retroreflected, and its frequency is tuned in order to maximize the blue MOT fluorescence.

Without the 497-nm repumper, the blue MOT lifetime is limited to $10 \mathrm{~ms}$ and typically we trap $10^{7}{ }^{88} \mathrm{Sr}$ atoms at 3 $\mathrm{mK}$. By applying the green repumper the blue MOT lifetime increases by more than one order of magnitude, resulting in up to $3 \times 10^{8}$ atoms loaded into the ${ }^{88} \mathrm{Sr}$ blue MOT at a density of $6 \times 10^{9} \mathrm{~cm}^{-3}$. These values are comparable to the alternative optical pumping scheme [22] in which two lasers at 707 and $679 \mathrm{~nm}$ couple the $5 p^{3} P_{2}$ and the $5 p^{3} P_{0}$ states to the $6 s^{3} S_{1}$ state (see Fig. 1).

As described in [1], the transfer from the blue to the red MOT is done in two steps: For the first $200 \mathrm{~ms}$ the red laser is frequency modulated at $50 \mathrm{kHz}$, spanning $2 \mathrm{MHz}$ (FM red MOT), to recapture the atoms moving faster than the capture velocity on the ${ }^{1} S_{0}-{ }^{3} P_{1}$ transition. In the following $40 \mathrm{~ms}$ the red laser is set single frequency at the intensity that determines the desired final temperature of the sample. Working at $350 \mathrm{kHz}$ below resonance and reducing the total light intensity on the MOT to $70 \mu \mathrm{W} / \mathrm{cm}^{2}$, we obtain about $10 \%$ of the initial atoms from the blue MOT cooled below $1 \mu \mathrm{K}$.

For simultaneous trapping of multiple isotopic samples previous experiments employed laser sources delivering the

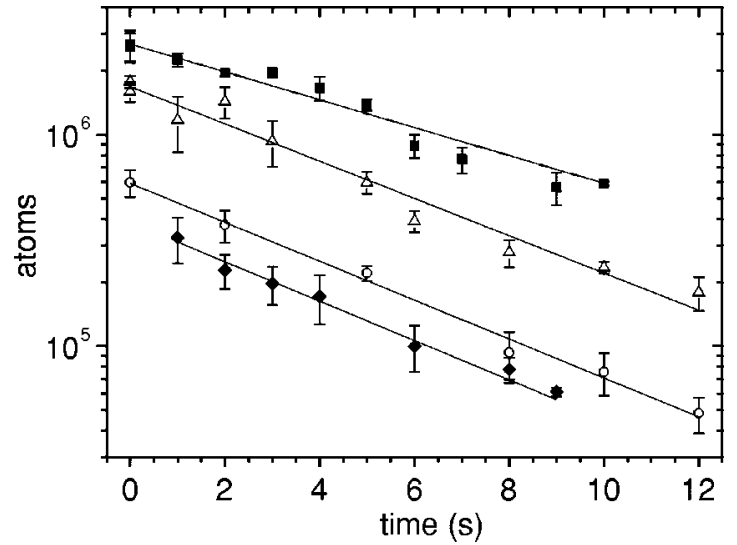

FIG. 2. Metastable ${ }^{3} P_{2}$ state decay when trapped in a $56-\mathrm{G} / \mathrm{cm}$ magnetic quadrupole. The data are taken for the individual isotopes with the ${ }^{1} S_{0^{-}}{ }^{1} P_{1}$ MOT switched off (circles: ${ }^{86} \mathrm{Sr}$, lifetime $\tau=4.7 \mathrm{~s}$. Squares: ${ }^{88} \mathrm{Sr}, \tau=6.6 \mathrm{~s}$ ), and with the ${ }^{1} S_{0^{-}}{ }^{1} P_{1}$ MOT operating on the undetected isotope (diamonds: ${ }^{86} \mathrm{Sr}$ with ${ }^{88} \mathrm{Sr}$ MOT, $\tau=4.7$ s. Triangles: ${ }^{88} \mathrm{Sr}$ with ${ }^{86} \mathrm{Sr}$ MOT, $\left.\tau=4.9 \mathrm{~s}\right)$. The measurements are taken after red MOT recapture and the $e^{-1}$ lifetimes are reported.

necessary frequency components for each isotope involved $[14,15]$. This approach in the case of the blue MOT may be difficult to apply because of the complexity of the laser sources, the limited optical access, the frequency offsets involved, and the limited laser power. An alterative solution is presented by the use of the magnetically trapped ${ }^{3} P_{2}$ state as a dark atom reservoir [23]. During the blue MOT phase without the repumper, the small branching ratio of the excited ${ }^{1} P_{1}$ state towards the metastable ${ }^{3} P_{2}$ state, provides a continuous loading of atoms into the potential given by the MOT's magnetic quadrupole [24]. Figure 2 reports the lifetime of the magnetically trapped metastable isotopes. Then, using the same blue source, one can sequentially load different isotopes into the magnetic potential just by stepping the laser frequency to the different resonances.

The trapping sequence for collection of ${ }^{88} \mathrm{Sr}^{8}{ }^{86} \mathrm{Sr}$ mixture is reported in Fig. 3. We start collecting ${ }^{86} \mathrm{Sr}$ for $3 \mathrm{~s}$, then we tune the blue laser on resonance to ${ }^{88} \mathrm{Sr}$ for $2 \mathrm{~s}$. Once the isotopic mixture is prepared in the ${ }^{3} P_{2}$ state, the blue light is switched off, and the FM red MOT is switched on as well as the repumping beam. The isotopic shift on the repumping

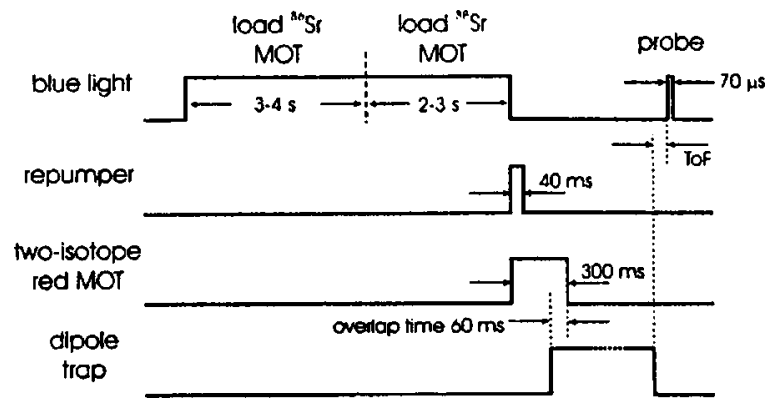

FIG. 3. Time sequence for simultaneous trapping of ${ }^{88} \mathrm{Sr}$ and ${ }^{86} \mathrm{Sr}$ in an optical dipole trap. MOT: magneto-optical trap. ToF: time of flight. 
transition is smaller than the resonance width of the ${ }^{3} P_{2^{-}}{ }^{3} D_{2}$ transition observed on the blue MOT fluorescence, which results in efficient, simultaneous optical pumping of ${ }^{88} \mathrm{Sr}$ and ${ }^{86} \mathrm{Sr}$ on a time scale short with respect to red MOT capture time [25]. The loading of a single isotope into the magnetic potential was already described in [24] and we did not observe significant differences in the behavior when loading two isotopes. Figure 2 shows the measurement of the lifetime for each isotope, both when individually trapped and in the presence of the blue MOT working on the other isotope. All the lifetimes are on the order of $5 \mathrm{~s}$, close to the background pressure limited lifetime of $8 \mathrm{~s}$.

The laser source for the operation of the two-isotope red MOT is composed of two slave lasers injected from the same frequency stabilized master with a frequency offset corresponding to the isotopic shift of $163817.3 \mathrm{kHz}$ [8]. Subsequently, the frequency and intensity of the two beams are controlled by double pass acusto-optic modulators driven by the same RF, the beams are superimposed on a polarizing beam splitter, and then they are overlapped to the blue MOT beams as described previously.

Comparing the two-isotope red MOT with the singleisotope one, with the same atom numbers we do not observe any effect in the transfer efficiency and final temperature due to the presence of the second isotope. In this way, we obtain samples with up to $10^{7}\left(10^{6}\right)$ atoms of ${ }^{88} \mathrm{Sr}\left({ }^{86} \mathrm{Sr}\right)$ at a temperature $2 \mu \mathrm{K}(1 \mu \mathrm{K})$. We attribute the difference in the loading with respect to the natural abundances to minor differences in the red MOT parameters. By varying the order of loading and the loading times of the two isotopes we can vary almost arbitrarily the final ratio of populations.

The cloud is then transferred into a FORT realized by focusing a far detuned 922-nm laser beam on the center of the trap. For this application we employ a semiconductor tapered amplifier seeded from the same 922-nm source used for the 461-nm laser. At this wavelength, setting the opticalfield polarization orthogonal to the magnetic field, the differential Stark shift between the ${ }^{1} S_{0}$ and ${ }^{3} P_{1}$ levels is small with respect to the transition linewidth, allowing precision spectroscopy and laser cooling even on optically confined atoms [26]. The light from the amplifier is spatially filtered through a polarization-maintaining single-mode optical fiber and is focused on the atoms to a waist of $15 \mu \mathrm{m}$. At maximum power, we measure the radial oscillation frequency $\nu_{\mathrm{rad}}=2 \mathrm{kHz}$ and the axial oscillation frequency $\nu_{\mathrm{ax}}=26 \mathrm{~Hz}$. These values are consistent with our peak intensity of $160 \mathrm{~kW} / \mathrm{cm}^{2}$, which corresponds to a trap depth of $90 \mu \mathrm{K}$.

As shown in Fig. 3, the transfer between the ${ }^{1} S_{0^{-}}{ }^{3} P_{1}$ MOT and the FORT is done by adding the 922-nm light 60 ms before switching off the MOT. Figure 4 shows the transfer of the mixture from the red MOT to the FORT as a function of the overlap time. The loading dynamics of ${ }^{86} \mathrm{Sr}$ is slightly faster than for ${ }^{88} \mathrm{Sr}$ while the losses at overlap time longer than $100 \mathrm{~ms}$ are attributed to the balance of lightassisted collisions [1]. The maximum transfer efficiency of $40 \%$ observed when the FORT beam passes through the MOT, with its waist displaced $500 \mu \mathrm{m}$ from the MOT center. This displacement corresponds to $2 / 3$ of Rayleigh range, or equivalently two red MOT $e^{-2}$ radii. The effects of this displacement are the increase of the overlap volume between

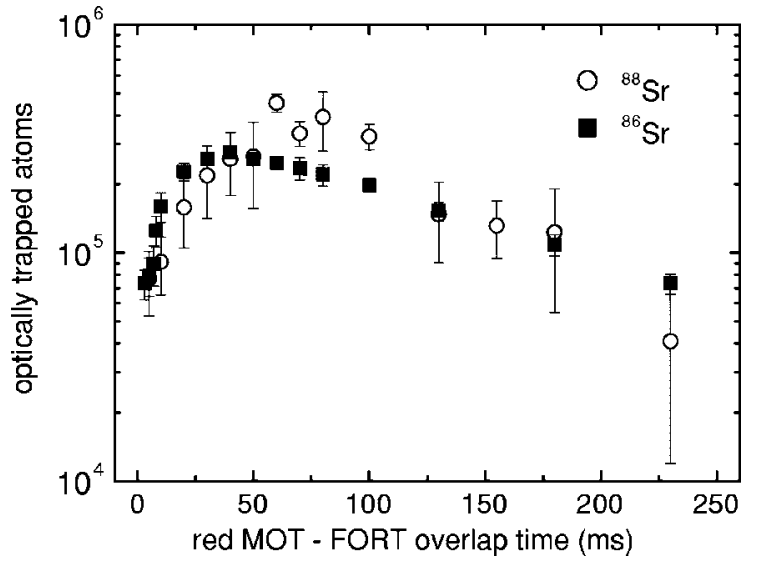

FIG. 4. Transfer from the intercombination red MOT to the optical dipole trap with different overlap time between the final red MOT and the FORT. The ${ }^{86} \mathrm{Sr}$ and ${ }^{88} \mathrm{Sr}$ mixture was prepared in order to load about twice ${ }^{88} \mathrm{Sr}$ than ${ }^{86} \mathrm{Sr}$ atoms in the final red MOT.

the final red MOT and the FORT, and the reduction of the light-assisted collisional losses at the bottom of the dipole potential. Finally, we load into the FORT up to 3 $\times 10^{5}\left(10^{6}\right)$ atoms typically at $15 \mu \mathrm{K}(20 \mu \mathrm{K})$ for the individual ${ }^{86} \mathrm{Sr}\left({ }^{88} \mathrm{Sr}\right)$ isotope. The difference in the final temperature is in agreement with the density-dependent heating reported in [1]. When loading the isotopic mixture we do not observe any reduction of the transfer efficiency from the red MOT into the FORT. Surprisingly in the optical trap we always find thermal equilibrium among the two species. The interspecies thermalization is fast on the time scale of the loading into the FORT and allows us to set a lower bound for the interspecies' elastic cross section $\sigma>3.5 \times 10^{-12} \mathrm{~cm}^{2}$. Equivalently, the modulus of the interspecies scattering length is $|a|=\sqrt{\sigma / 8 \pi}>70$ Bohr radii.

In conclusion, we presented the simultaneous cooling and trapping of the ${ }^{88} \mathrm{Sr}$ and ${ }^{86} \mathrm{Sr}$ isotopes into a MOT operating on the spin-forbidden ${ }^{1} S_{0^{-}}{ }^{3} P_{1}$ transition, and we described the transfer of the mixture into a conservative, far-offresonant, dipole trap working near the "magic wavelength" for the ${ }^{1} S_{0^{-}}{ }^{3} P_{1}$ transition [26]. The method, which is demonstrated with strontium isotopes, can be applied to all the atoms with similar level structure and transitions, to load an arbitrarily large number of isotopes. The realization of the strontium isotopic mixture and the observed strong interspecies thermalization will allow the implementation of additional cooling mechanisms based on sympathetic cooling. This seems very promising towards the realization of a BoseEinstein condensate of an alkali-earth-metal atom.

We acknowledge fruitful discussions with M. Prevedelli, J. L. Hall, and C. Salomon, and contributions in the early stages of the experiment from P. Roulleau. We thank R. Ballerini, M. De Pas, M. Giuntini, and A. Hajeb for technical assistance. J.L. acknowledges support from the EU network on Cold Quantum Gases (HPRN-CT-2000-00125). This work was supported by Agenzia Spaziale Italiana, Ente Cassa di Risparmio di Firenze, MIUR-COFIN 2002, Istituto Nazionale Fisica della Materia, Istituto Nazionale di Fisica Nucleare, and LENS. 
[1] H. Katori, T. Ido, Y. Isoya, and M. Kuwata-Gonokami, Phys. Rev. Lett. 82, 1116 (1999).

[2] T. Mukaiyama, H. Katori, T. Ido, Y. Li, and M. KuwataGonokami, Phys. Rev. Lett. 90, 113002 (2003).

[3] X. Xu, T. H. Loftus, J. W. Dunn, C. H. Greene, J. L. Hall, A. Gallagher, and J. Ye, Phys. Rev. Lett. 90, 193002 (2003).

[4] T. H. Loftus, T. Ido, A. D. Ludlow, M. M. Boyd, and J. Ye, Phys. Rev. Lett. 93, 073003 (2004).

[5] H. Katori, T. Ido, and M. Kuwata-Gonokami, in Atomic Physics 17, edited by E. Arimondo, P. de Natale, and M. Inguscio, AIP Conf. Proc. No. 551 (AIP, New York: Melville, 2001), p. 382.

[6] I. Courtillot, A. Quessada, R. P. Kovacich, A. Brusch, D. Kolker, J.-J. Zondy, G. D. Rovera, and P. Lemonde, Phys. Rev. A 68, $030501(\mathrm{R})$ (2003).

[7] M. Takamoto and H. Katori, Phys. Rev. Lett. 91, 223001 (2003)

[8] G. Ferrari, P. Cancio, R. Drullinger, G. Giusfredi, N. Poli, M. Prevedelli, C. Toninelli, and G. M. Tino, Phys. Rev. Lett. 91, 243002 (2003).

[9] T. Ido, T. H. Loftus, M. M. Boyd, A. D. Ludlow, K. W. Holman, and J. Ye, Phys. Rev. Lett. 94, 153001 (2005).

[10] Y. Bidel, B. Klappauf, J. C. Bernard, D. Delande, G. Labeyrie, C. Miniatura, D. Wilkowski, and R. Kaiser, Phys. Rev. Lett. 88, 203902 (2002).

[11] A. Derevianko, S. G. Porsev, S. Kotochigova, E. Tiesinga, and P. S. Julienne, Phys. Rev. Lett. 90, 063002 (2003).

[12] T. Ido, Y. Isoya, and H. Katori, Phys. Rev. A 61, 061403(R) (2000).

[13] M. S. Santos, P. Nussenzveig, L. G. Marcassa, K. Helmerson, J. Flemming, S. C. Zilio, and V. S. Bagnato, Phys. Rev. A 52, R4340 (1995).

[14] W. Suptitz, G. Wokurka, F. Strauch, P. Kohns, and W. Ertmer,
Opt. Lett. 19, 1571 (1994).

[15] M.-O. Mewes, G. Ferrari, F. Schreck, A. Sinatra, and C. Salomon, Phys. Rev. A 61, 011403(R) (1999).

[16] R. E. Drullinger, D. J. Wineland, and J. C. Bergquist, Appl. Phys. 22, 365 (1980).

[17] E. L. Raab, M. Prentiss, A. Cable, S. Chu, and D. E. Pritchard, Phys. Rev. Lett. 59, 2631 (1987).

[18] F. Shimizu, K. Shimizu, and H. Takuma, Chem. Phys. 145, 327 (1990).

[19] W. D. Phillips and H. Metcalf, Phys. Rev. Lett. 48, 596 (1982).

[20] G. Ferrari, T. Brzozowski, R. Drullinger, N. Poli, M. Prevedelli, C. Toninelli, and G. M. Tino, Proc. SPIE 5478, 210 (2004).

[21] The cell, $60 \mathrm{~cm}$ long and $2 \mathrm{~cm}$ in diameter, looks quite similar to a heatpipe with heating in the central region, water cooling at the windows, and argon as buffer gas to limit the diffusion of strontium vapor to the windows. The central part is heated to $380{ }^{\circ} \mathrm{C}, 400{ }^{\circ} \mathrm{C}$ less than the strontium melting point. Even though the system does not work in the heatpipe regime, we could operate it longer than six months uninterruptedly.

[22] T. P. Dinneen, K. R. Vogel, E. Arimondo, J. L. Hall, and A. Gallagher, Phys. Rev. A 59, 1216 (1999).

[23] J. Stuhler, P. O. Schmidt, S. Hensler, J. Werner, J. Mlynek, and T. Pfau, Phys. Rev. A 64, 031405(R) (2001).

[24] S. B. Nagel, C. E. Simien, S. Laha, P. Gupta, V. S. Ashoka, and T. C. Killian, Phys. Rev. A 67, 011401(R) (2003).

[25] Since the optical pumping is based on the scattering of few photons, in case of larger isotopic shift simultaneous optical pumping from the ${ }^{3} P_{2}$ state can be recovered by frequency modulating the repumper laser on the suited interval.

[26] T. Ido and H. Katori, Phys. Rev. Lett. 91, 053001 (2003). 